\title{
ON THE SEMIGROUP OF HADAMARD DIFFERENTIABLE MAPPINGS
}

\author{
SADAYUKI YAMAMURO
}

(Received 26 October 1970)

Communicated by J. B. Miller

The main purpose of this paper is to prove that every automorphism of the semigroup of all Hadamard-differentiable mappings of a separable real Banach space into itself is inner. This generalizes the result of [7] which is a generalization of a result proved by Magill, Jr. [5].

We start with a brief account on the Hadamard differentiation.

\section{The Hadamard differentiation}

The following method of defining derivatives has been given by Averbukh and Smolyanov $[1,2]$, where it was also proved that the Hadamard differentiability defined below is equivalent to the quasi-differentiability defined by Dieudonné $[3$, p. 151$]$.

Let $E$ be a real Banach space, and let $M$ be a class of some subsets of $E$. We denote by $\mathscr{L}=\mathscr{L}(E)$ the Banach algebra of all continuous linear mappings of $E$ into itself with the usual algebraic structure and the upper bound norm. Then, a mapping $f: E \rightarrow E$ is said to be $M$-differentiable at $a \in E$ if there exists $u \in \mathscr{L}$ such that, for any $M \in M$,

$$
\sup _{x \in M}\left\|\varepsilon^{-1} r(f, a, \varepsilon x)\right\| \rightarrow 0 \text { if } \varepsilon \rightarrow 0,
$$

where

$$
r(f, a, x)=f(a+x)-f(a)-u(x) .
$$

We denote by $\mathscr{D}_{M}$ the set of all $f: E \rightarrow E$ which are $M$-differentiable at every point of $E$.

(1) If $M$ is the class of all single point sets, then $f \in \mathscr{D}_{M}$ is said to be Gatteauxdifferentiable. In this case, we denote $\mathscr{D}_{M}$ by $\mathscr{D}_{G}$.

(2) If $M$ is the class of all compact subsets, then $f \in \mathscr{D}_{M}$ is said to be Hadamard-differentiable. In this case, we denote $\mathscr{D}_{M}$ by $\mathscr{D}_{H}$.

(3) If $M$ is the class of all bounded subsets, then $f \in \mathscr{D}_{M}$ is said to be Fréchetdifferentiable. In this case, we denote $\mathscr{D}_{M}$ by $\mathscr{D}_{F}$. 
In each of these cases, the continuous linear mapping $u$ is determined uniquely and is denoted by $f^{\prime}(a)$. It is called the Gâteaux-, the Hadmarad-or the Fréchetderivative of $f$ at a respectively.

Obviously,

$$
\mathscr{L} \subset \mathscr{D}_{F} \subset \mathscr{D}_{H} \subset \mathscr{D}_{G},
$$

and the inclusions are generally strict. If $E$ is finite-dimensional, we have $\mathscr{D}_{F}=\mathscr{D}_{G}$, and if $E$ is one dimensional we have $\mathscr{D}_{F}=\mathscr{D}_{H}=\mathscr{D}_{G}$.

The following theorem will be used in the following section. We shall denote by $\mathscr{K}$ the set of all completely continuous (i.e,. continuous and compact) mapping of $E$ into itself. We also denote by $\mathscr{K}_{1}$ the set of all $f: E \rightarrow E$ such that

$$
f(x)=\mu(\langle x, \bar{a}\rangle) \text { for every } x \in E,
$$

where $\mu$ is any differentiable $E$-valued function of a real variable, $\bar{a} \in \bar{E}$ (the conjugate space of $E$ and $\langle x, \bar{a}\rangle$ is the value of $\bar{a}$ at $x$. Obviously, $\mathscr{K}_{1} \subset \mathscr{K}$.

In the sequel, the composition of two mappings $f, g: E \rightarrow E$ is denoted by $f g$ that is,

$$
(f g)(x)=f(g(x)) \text { for every } x \in E .
$$

THEOREM 1. 1) If $f \in \mathscr{D}_{H}$, then $f k \in \mathscr{D}_{F}$ for any $k \in \mathscr{D}_{F} \cap \mathscr{K}$ and

$$
(f k)^{\prime}(a)=f^{\prime}(k(a)) k^{\prime}(a) \text { for any } a \in E \text {. }
$$

2) If $f \in \mathscr{D}_{G}$, and if for every $k \in \mathscr{D}_{F} \cap \mathscr{K}_{1}$ it is true that $f k \in \mathscr{D}_{G}$ and $\left({ }^{*}\right)$ is satisfied, then $f \in \mathscr{D}_{H}$.

PRoOF. 1) For $f \in \mathscr{D}_{H}$ and $k \in \mathscr{D}_{F} \cap \mathscr{K}$,

$$
\begin{gathered}
f k(a+x)-f k(a)-f^{\prime}(k(a)) k^{\prime}(a)(x) \\
=f^{\prime}(k(a))[k(a+x)-k(a)]+r(f, k(a), k(a+x)-k(a))- \\
-f^{\prime}(k(a)) k^{\prime}(a)(x) \\
=f^{\prime}(k(a)) r(k, a, x)+r(f, k(a), k(a+x)-k(a)) .
\end{gathered}
$$

Then, for a bounded set $B$, since $k \in \mathscr{D}_{F}$,

and

$$
\begin{aligned}
& \sup _{x \in B}\left\|\varepsilon^{-1} f^{\prime}(k(a)) r(k, a, \varepsilon x)\right\| \\
& \leqq\left\|f^{\prime}(k(a))\right\| \sup _{x \in B}\left\|\varepsilon^{-1} r(k, a, \varepsilon x)\right\| \rightarrow 0 \quad \text { if } \varepsilon \rightarrow 0,
\end{aligned}
$$

$$
\begin{aligned}
\sup _{x \in B} & \left\|\varepsilon^{-1} r(f, k(a), k(a+\varepsilon x)-k(a))\right\| \\
& =\sup _{x \in B}\left\|\varepsilon^{-1} r\left(f, k(a), \varepsilon\left[\varepsilon^{-1}(k(a+\varepsilon x)-k(a))\right]\right)\right\| \rightarrow 0 \quad \text { if } \varepsilon \rightarrow 0,
\end{aligned}
$$

because, since $k \in \mathscr{K}$, for any $\varepsilon_{n} \rightarrow 0$, the set 


$$
\left\{\varepsilon_{n}^{-1}\left(k\left(a+\varepsilon_{n} x\right)-k(a)\right) \mid x \in B, \quad n=1,2, \cdots\right\}
$$

is contained in a compact set. In fact, since

$$
\varepsilon_{n}^{-1}\left(k\left(a+\varepsilon_{n} x\right)-k(a)\right)=k^{\prime}(a)(x)+\varepsilon_{n}^{-1} r\left(k, a, \varepsilon_{n} x\right),
$$

the fact that $k^{\prime}(a) \in \mathscr{K}\left(\left[6\right.\right.$, p. 27]) implies that $\left\{k^{\prime}(a)(x) \mid x \in B\right\}$ is contained in a compact set and the fact that $k \in \mathscr{D}_{F}$ implies that the second term converges to 0 as $n \rightarrow \infty$. Therefore,

$$
f k \in \mathscr{D}_{F} \text { and }(f k)^{\prime}(a)=f^{\prime}(k(a)) k^{\prime}(a) .
$$

2) Let us assume that $f \notin \mathscr{D}_{H}$. Then, there exist $\varepsilon_{n} \downarrow 0, a \in E$ and $x_{n} \rightarrow x_{0}$ such that

$$
\varepsilon_{n}^{-1} r\left(f, a, \varepsilon_{n} x_{n}\right) \nrightarrow 0 \text { as } n \rightarrow \infty .
$$

Now, the method used in $[2, \mathrm{~s}, 92]$ supplies a differentiable $E$-valued function $\mu(\xi)$ of a real variable such that

$$
\mu(0)=a, \quad \mu\left(\varepsilon_{n}\right)=a+\varepsilon_{n} x_{n} \text { and } \mu^{\prime}(0)=x_{0} .
$$

Then, consider the mapping $k \in \mathscr{K}_{1}$ defined by

$$
k(x)=\mu(\langle x, \bar{a}\rangle),
$$

where $\vec{a} \in \bar{E}$ and $\langle a, \bar{a}\rangle=1$. By the assumption,

$$
f k \in \mathscr{D}_{G} \quad \text { and } \quad(f k)^{\prime}(0)=f^{\prime}(k(0)) k^{\prime}(0) .
$$

On the other hand,

$$
\begin{aligned}
k^{\prime}(0)(a) & =\lim _{\varepsilon \rightarrow 0} \varepsilon^{-1}[k(\varepsilon a)-k(0)] \\
& =\lim _{\varepsilon \rightarrow 0} \varepsilon^{-1}[\mu(\varepsilon)-\mu(0)]=\mu^{\prime}(0)=x_{0},
\end{aligned}
$$

and

$$
\begin{array}{rl}
\varepsilon_{n}^{-1} & r\left(f, a, \varepsilon_{n} x_{n}\right) \\
& =\varepsilon_{n}^{-1}\left[f\left(a+\varepsilon_{n} x_{n}\right)-f(a)-f^{\prime}(a)\left(\varepsilon_{n} x_{n}\right)\right] \\
& =\varepsilon_{n}^{-1}\left[f k\left(\varepsilon_{n} a\right)-f k(0)-(f k)^{\prime}(0)\left(\varepsilon_{n} a\right)\right]+(f k)^{\prime}(0)(a)-f^{\prime}(a)\left(x_{n}\right) \\
& =\varepsilon_{n}^{-1} r\left(f k, 0, \varepsilon_{n} a\right)+f^{\prime}(a)\left(x_{0}-x_{n}\right) \rightarrow 0 \text { if } n \rightarrow \infty,
\end{array}
$$

which is a contradiction.

\section{2. $\mathscr{D}_{H}$ as a semigroup}

It is well-known that if $f, g \in \mathscr{D}_{F}$ then $f g \in \mathscr{D}_{F}$. In other words, $\mathscr{D}_{F}$ is a semigroup with respect to the composition. For any semigroup $\mathscr{D}$, a one-to-one mapping $\phi$ of $\mathscr{D}$ onto itself is called an automorphism if 


$$
\phi(f g)=\phi(f) \phi(g) \text { for } f, g \in \mathscr{D} \text {. }
$$

If there exists $h \in \mathscr{D}$ such that it has the two-sided inverse $h^{-1}$ in $\mathscr{D}$ and

$$
\phi(f)=h f h^{-1} \text { for every } f \in \mathscr{D}
$$

then the automorphism is said to be inner.

Eidelheit [4] has proved that every continuous automorphism of the semigroup $\mathscr{L}$ is inner.

On the other hand, Magill, Jr. [5] has proved that, if $E$ is one-dimensional, every automorphism of the semigroup $\mathscr{D}_{F}\left(=\mathscr{D}_{H}=\mathscr{D}_{G}\right)$ is inner.

These two results take us naturally to the question whether every automorphism of the semigroup $\mathscr{D}_{F}$ on a general Banach space is inner.

Eidelhiet's result suggests that we may need some continuity assumptions. In fact, in [9], we have shown that, in the semigroup of all boundely and continuously differentiable mappings, where the topology is defined by

$$
\|f\|_{n}=\sup _{\|x\| \leqq n}\left\{\|f(x)\|+\left\|f^{\prime}(x)\right\|\right\}, \quad n=1,2, \cdots,
$$

an automorphism is inner if and only if it is continuous.

On the other hand, in [8] we have given a necessary and sufficient condition for an automorphism $\phi$ of $\mathscr{Z}_{F}$ to be inner. The method used there has been refined in [7], where we have generalized the Magill's result mentioned above to arbitrary finite-dimensional Banach spaces.

Now, we turn to the set $\mathscr{D}_{H}$. As Averbukh and Smolyanov $[1,2]$ have pointed out, the Hadamard differentiation is, in a sense, the weakest differentiation which has the composition property: if $f, g \in \mathscr{D}_{H}$, then $f g \in \mathscr{D}_{H}$ and

$$
(f g)^{\prime}(a)=f^{\prime}(g(a)) g^{\prime}(a) \text { for every } a \in E .
$$

Moreover, if $E$ is finite-dimensional, then $\mathscr{D}_{F}=\mathscr{D}_{H}$. Therefore, the following result is a generalization of that of [7]:

THEOREM 2. Let E be separable. Then, every automorphism of the semigroup $\mathscr{D}_{\boldsymbol{H}}$ is inner.

Proof. Let $\phi$ be an automorphism. Exactly the same argument as in [7], if $\mathscr{D}_{F}$ there is replaced by $\mathscr{D}_{H}$, gives the following facts:

(1) there exists a unique one-to-one mapping $h$ of $E$ onto $E$ such that

$$
\phi(f)=h f h^{-1} \text { for every } f \in \mathscr{D}_{H} .
$$

(2) $h \in \mathscr{D}_{G}$ and $h^{-1} \in \mathscr{D}_{G}$;

(3) $(a \otimes \bar{a}) h \in \mathscr{D}_{H}$ for any $a \in E$ and $\bar{a} \in \bar{E}$, where $a \otimes \bar{a}$ is an element of $\mathscr{L}$ defined by

$$
(a \otimes \bar{a})(x)=\langle x, \bar{a}\rangle a \quad \text { for every } \quad x \in E
$$


and

(4) $((a \otimes \bar{a}) h)^{\prime}(x)(y)=\left\langle h^{\prime}(x)(y), \bar{a}\right\rangle a$

We shall prove that $h \in \mathscr{D}_{H}$. Since we may start with $\phi^{-1}$ instead of $\phi$, we use the fact that any result containing $h$ remains true if we replace $h$ by $h^{-1}$.

Now, by Theorem 1, we have only to prove that

$$
h k_{1} \in \mathscr{D}_{G} \text { for any } k_{1} \in \mathscr{K}_{1} \cap \mathscr{D}_{F}
$$

and

$$
\left(h k_{1}\right)^{\prime}(x)=h^{\prime}\left(k_{1}(x)\right) k_{1}^{\prime}(x) .
$$

Let us take an arbitrary $k_{1} \in \mathscr{K}_{1}$ :

$$
k_{1}(x)=\mu(\langle x, \bar{a}\rangle),
$$

and let $a \in E$ be such that $\langle a, \bar{a}\rangle=1$. Then, we have $k_{1}=k_{1}(a \otimes \bar{a})$. Since Since $a \otimes \bar{a} \in \mathscr{L} \subset \mathscr{D}_{H}$, there exists $k \in \mathscr{D}_{H}$ such that

Since

$$
\phi(k)=a \otimes \bar{a} .
$$

$$
k(x)=h^{-1}(\langle h(x), \bar{a}\rangle a),
$$

where $\langle h(x), \bar{a}\rangle$ is continuous by $[8, \mathrm{p} .506]$ and $h^{-1}(\xi a)$ is continuous with respect to $\xi$ by (2) above, we see that $k \in \mathscr{K}$. Therefore, from (3) it follows that

$$
(a \otimes \bar{a}) h k \in \mathscr{D}_{\boldsymbol{H}} \cdot
$$

Since

$$
(a \otimes \bar{a}) h k(x)=\langle h k(x), \bar{a}\rangle a,
$$

the mapping $\langle h k(x), \bar{a}\rangle$ of $E$ into the set of real numbers is Hadamard-differentiable. Therefore, the mapping $\mu(\langle h k(x), \bar{a}\rangle)$ of $E$ into $E$ is Hadamard-differentiable and obviously,

$$
\mu(\langle h k(x), \bar{a}\rangle)=k_{1} h k(x) \text { for every } x \in E .
$$

In other words,

$$
k_{1} h k \in \mathscr{D}_{\boldsymbol{H}}
$$

Therefore,

and

$$
\phi\left(k_{1} h k\right) \in \mathscr{D}_{\boldsymbol{H}}
$$

$$
\phi\left(k_{1} h k\right)=h k_{1} h k h^{-1}=h k_{1} \phi(k)=h k_{1}(a \otimes \bar{a})=h k_{1},
$$

from which it follows that

$$
h k_{1} \in \mathscr{D}_{\boldsymbol{H}}
$$


Thus, it only remains to prove the equality $\left(^{*}\right)$ of Theorem 1 . First, since $a \otimes \bar{a} \in \mathscr{D}_{H}$ and $h k_{1} \in \mathscr{D}_{H}$, we have

$$
(a \otimes \bar{a}) h k_{1} \in \mathscr{D}_{H} \text { and }\left((a \otimes \bar{a}) h k_{1}\right)^{\prime}(x)(y)=\left\langle\left(h k_{1}\right)^{\prime}(x)(y), \bar{a}\right\rangle a .
$$

Also, by applying Theorem 1,1$)$ to $(a \otimes \bar{a}) h$ and $k_{1}$ we have

$$
\left((a \otimes \bar{a}) h k_{1}\right)^{\prime}(x)(y)=((a \otimes \bar{a}) h)^{\prime}\left(k_{1}(x)\right) k_{1}^{\prime}(x)(y)
$$

and by (3) and (4) the right hand side here is $\left\langle h^{\prime}\left(k_{1}(x)\right) k_{1}^{\prime}(x)(y), \bar{a}\right\rangle a$. Therefore,

$$
\left\langle\left(h k_{1}\right)^{\prime}(x)(y), \bar{a}\right\rangle a=\left\langle h^{\prime}\left(k_{1}(x)\right) k_{1}^{\prime}(x)(y), \bar{a}\right\rangle a .
$$

Since $\vec{a}$ is arbitrary, $\left({ }^{*}\right)$ follows, Thus. $h \in \mathscr{D}_{H}$ and hence $\phi$ is inner.

REMARK. With the product defined above and the addition $f+g$ defined by

$$
(f+g)(x)=f(x)+g(x) \text { for every } x \in E,
$$

$\mathscr{D}_{H}$ is a near-ring. The fact that every near-ring automorphism of $\mathscr{D}_{H}$ is inner can be proved in the same way as in [9]. In this case, $h$ is in $\mathscr{L}$.

\section{References}

[1] V. I. Averbukh and O. G. Smolyanov, 'The theory of differentiation in linear topological spaces,' Russian Math. Survey, 22 : 6 (1967), 201-258.

[2] V. I. Averbukh and O. G. Smolyanov, 'The various definitions of the derivative in linear topological spaces,' Russian Math. Survey, 23: 4 (1968), 67-113.

[3] J. Dieudonné, Foundations of Modern Analysis (1960).

[4] M. Eidelheit, 'On isomorphisms of rings of linear operators.' Studia M. 9 (1940), 97-105.

[5] K. D. Magill, Jr., 'Automorphisms of the semigroup of all differentiable functions,' Glasgow Math. J. 8 (1967), 63-66.

[6] J. T. Schwartz, Non-linear Functional Analysis (Gordon and Breach, New York, 1969).

[7] G. R. Wood and Sadayuki Yamamuro, 'On the semigroup of differentiable mappings' (II); (to appear in Glasgow Math. J.).

[8] Sadayuki Yamamuro, 'On the semigroup of differentiable mappings,'J. Australian Math. Soc. 10 (1969), 503-510.

[9] Sadayuki Yamamuro, 'On the semigroup of bounded $C^{1}$-mappings,' (to appear in J. Australian Math. Soc.)

Department of Mathematics

Institute of Advanced Studies

Australian National University 